\title{
Biodiversity in Urban environments of Ordu City and Nearby Areas: Mammals, Birds, Reptiles and Amphibians
}

\section{Ordu Şehri ve Yakın Çevresinde Biyoçeşitlik: Memeliler, Kuşlar, Sürüngenler ve İkiyaşamlılar}

\author{
Research Article
}

Hasan Sevgilii', Ahmet Karataş², Onur Candan'

'Ordu University, Faculty of Art and Science, Department of Biology, Ordu.

${ }^{2}$ Niğde University, Faculty of Art and Science, Department of Biology, Niğde.

\begin{abstract}
A B S TR AC T
The present paper provides the first species list of the fauna of terrestrial vertebrates of Ordu, Turkey. A survey for determination of the fauna was conducted between 2011 and 2015 in Ordu and the immediate vicinity. The field studies were made using several different techniques including; visual, wildlife photography and passive infrared camera traps. Previous records and wildlife observations around Ordu city published by professional wildlife photographers were also included. Overall, we determined 219 terrestrial vertebrate species (168 birds, 40 mammals, 7 reptiles, and 4 amphibians), some of them being low conservation concern. Among the most remarkable species from the conservation point of view, Emys orbicularis (Reptilia), Aythya nyroca, Puffinus yelkouan, Pelecanus crispus, Saxicola maurus, Tetrax tetrax (Aves), Rhinolophus euryale, Miniopterus schreibersii, Lutra lutra (Mammalia) come into prominence. The third record of the Rustic Bunting (Emberiza rustica) for Turkey is made in this study. In the Blacksea region of Turkey, although the most records of Roe Deer (Capreolus capreolus) is largely confined to mountainous regions, it was captured by the photo-traps in the urban areas. Our results suggest that biodiversity of terrestrial vertebrates in Ordu and immediate vicinity is still rich, and adjacent environments between urban and wild habitats are important in terms of conservation of the urban biodiversity.
\end{abstract}

\section{Key Words}

Terrestrial Fauna, mammals, birds, herptiles, Ordu province, Turkey.

\section{ÖZET}

$\mathrm{B}$ çalışmada Ordu ilinin karasal omurgalı türleri ilk kez listelenmiştir. Arazi çalışması Ordu şehri ve yakın çevresinde 2011-2015 yılları arasında gerçekleştirilmiştir. Arazi çalışmaları doğrudan gözlem, fotoğraflama, fotokapan ve video kullanılarak farklı yöntemlerle gerçekleştirilmiştir. Tür listesi Ordu şehri ve civarında yapılmış çalışmalardan elde edilen kayıtlar ve profesyonel doğa fotoğrafçılarının gözlemlerini de yansıtmaktadır. Genel olarak, toplamda 219 adet karasal omurgalı türü (168 kuş, 40 memeli, 7 sürüngen, 4 kurbağa) tespit edilmiş ve bunların çok az bir kısmının tehlike altında olduğu anlaşılmaktadır. Koruma statüleri bakımından dikkat çeken türler olarak; Emys orbicularis, Aythya nyroca, Puffinus yelkouan, Pelecanus crispus, Saxicola maurus, Tetrax tetrax, Rhinolophus euryale, Miniopterus schreibersii, Lutra lutra verilebilir. Akkaşlı Kirazkuşunun (Emberiza rustica), Türkiye'den 3. kaydı bu çalışma ile verilmektedir. Karadeniz bölgesinde Karaca (Capreolus capreolus)'nın birçok kaydının daha çok dağlık bölgelerle sınırlı kalmasına rağmen, bu çalışmada şehirleşen alanda da fotokapan ile tespit edilmiştir. Bu çalışmadan elde edilen sonuçlar, Ordu şehri ve yakın civarının karasal omurgalı çeşitliliğinin hala yüksek olduğu ve şehir ile doğal yaşam habitatları arasındaki çevrelerin şehir biyoçeşitliliğinin korunmasında önemli olduğuna işaret etmektedir.

\section{Anahtar Kelimeler}

Karasal Fauna, Memeli, Kuş, Herptil, Ordu şehri, Türkiye.

Article History: Received: Jan 7, 2016; Revised: Feb 10, 2016; Accepted: Mar 12, 2016; Available Online: Apr $01,2016$.

DOI: $10.15671 /$ HJBC.20164417566

Correspondence to: H. Sevgili; Ordu University, Faculty of Art and Science, Department of Biology, Ordu, Turkey. 


\section{INTRODUCTION}

A S of 2014, it is estimated that $54 \%$ of the world population live in cities, and this rate will rise up to $66 \%$ by 2050 [1]. According to the address-based population registration system data, $23 \%$ of the population of Turkey lives in rural areas, whereas the remaining $77 \%$ lives in urban areas [2]. A modification of the natural distribution areas of organisms into farmlands, urbanization and growth of the human population have a strong impact on biodiversity $[3,4]$. The migration from the countryside to city engenders an utilization of new lands for housing, resulting in a separation of the present habitats into a fragmented structure or a complete eradication of the ecosystem. There are three main aspects of biodiversity: species diversity, genetic diversity and ecosystem diversity. A devastation of the habitats is considered as "habitat loss" for many species, which results in a pollution, infestation of invasive species and other adversities. In conclusion, of the increase in the urbanization rate, and exploitation of land for agriculture appear to be the main threats of continuity of local species $[5,6]$.

This study aimed to determine the terrestrial vertebrates observed in Ordu and the immediate vicinity. Ordu city was established in 400 BC by the name Kotyora [7]. Residential life quickly formed after the establishment of the Republic of Turkey. Means of livelihood include traditional fishing and, hazelnut cultivation that has been recently popularized [8]. In mid 1960s, 15\% of the population had lived in cities while this rate increased to $45 \%$ in 2012.

As known, the East and the West Black Sea geographical regions are separated by the river Melet. Until recent years, the river basins; Durugöl, Melet, and Turnasuyu have been intact habitats, as understood from the records. The geological formation of the River Basin, Melet, indicates the presence of these natural habitats [9]. According to Gürgen [9], powerful streams such as Melet, Civil, and Turnasuyu generated the delta formed from the vast Pleistocene and Holocene alluvial fillings on the coasts in the west of Boztepe. However, after 1950 these basins and wetlands were opened for settlement, dried to create agricultural fields in particular, habitats of birds and other terrestrial vertebrates were rapidly devastated. In addition to increasing urbanization, the usage of the estuary of Melet as garbage dump for many years remarkably polluted the area. In addition, the bird species visiting the area had significantly dropped. Our observations in a small wetland which has been able to still survive, but partly-impaired and polluted in Durugöl, which is totally occupied by the buildings today showed that the water bird species (Anatidae, water hens, herons etc.), hinting that the species richness of the area in the period which the wetland was not polluted. As it can be seen in the study results, an observation of mammals like Roe Deer (Capreolus capreolus) near urban areas, supports this thesis.

Turkey, when compared to other Palearctic geographical regions, has a richer habitat variety. The fact that endemic taxa in a proportional sense is higher in comparison with other countries indicates that terrestrial, nautical, and freshwater biodiversity is greater [6]. Turkey's avifauna like mammal and reptile faunas have been thoroughly studied, and a precise list is mostly established. It is also known that Turkey's avifauna mostly have been photographed in the web sources created by professional and amateur photographers [10, 11]. Kirwan et al. [12] have compiled and listed numerous studies concerning with Turkey's bird fauna, and reported 453 species. It has been determined that most of these bird species is commonly found throughout the year and very few of them (approximately 56) are migratory birds [13]. Later studies [14] also confirmed that approximately 460 species were reported from Turkey. The list of birds in Turkey have been updated, and with the recent records, the list contains 483 species $[10,15]$.

The Kızılırmak Delta (Samsun), which is the closest habitat to the presently studied area as well as the best preserved and biggest wetland in the Blacksea Region, contains more than 340 determined bird species [16]. In a study done in Artvin, situated on a bird migration route, a total of 216 bird species including 87 local species was determined [17]. Except for a few records, there is no avifauna studies in Ordu and 
immediate vicinity. However, in a report prepared by the Provincial Directorate of Environment of Ordu, a list of mammal, bird and reptile species determined in Ordu is provided [18]. According to this list, 62 birds, 19 reptiles and 31 mammals species were listed in Ordu. Interestingly, the same list was repeated in 2013.

A study listing Turkey and Cyprus's mammal fauna reviewed the studies on mammal fauna in Turkey and listed over 140 species [19]. According to the list, a significant amount of mammal species in Turkey consists of rodents with $43 \%$, while $24 \%$ consists of bats, $13,5 \%$ carnivores, and $12 \%$ insectivores. Devastation of habitats and the rapid urbanization negatively effects not only bird faunas, but also mammal faunas $[20,21]$. In the present study, since large mammal observations rather than small mammals were made around the university campus with phototrap method, potential small mammals, and bats were not determined.

As noted above, Turkey also has a herpetofauna that is quite diverse in terms of reptile (Reptilia) and amphibian (Amphibia) groups due to different geographic areas and an intercontinental bridge. Numerous studies have reported a rapid decrease in the distribution and abundance of reptiles and amphibians after the second half of the 20th century $[22,23]$. Despite the fact that a consensus on taxa have not been reached in terms of species status, there are 21 species of amphibians, 8 of them are salamenders (urodela), and 13 of them are frogs (Anura) in Turkey [24]. In the same book, a total of 108 reptile species including 9 tortoises, 58 lizards, and 41 serpentes were listed. In our country, reptile observation and photography and relevant science-based internet sources have recently started to become common. According to the data of Anonymous [25], one of the most important of the sources, a total of 34 amphibian species including 17 salamenders (Urodela), and 17 frogs (Anura), and a total of 132 reptile species including 11 tortoises and 65 lizards, 56 serpentes have been listed. The reasons of different number of species from the Baran's list [24] are the addition of new taxa in the list or other taxonomic changes. Except for some local faunistic studies related to the Black
Sea region [e.g.26], a complete herpetofauna list has not been established yet. According to the results of the only herpetofaunistic study to include Ordu and Giresun provinces in the study area, with 3 salamenders (Urodela), 6 frogs (Anura), and 4 lizards, 4 serpentes, a total of 17 species were determined (Kumlutaş et al., 1998). A study in Middle and East Black Sea regions reported 7 amphibians, 2 tortoises, 12 lizards, and 5 serpentes species $[27,28]$.

\section{MATERIAL AND METHOD}

This study includes data collected irregularly between 2011-2015. The bird species in the list were determined in Cumhuriyet campus and the immediate vicinity of Ordu University, Melet River estuary, inner-city, coastal region, and Turnasuyu estuary. The birds were determined in all seasons by photography and observations irregular done in irregular intervals. Photography was done using Canon EOS-1D Mark IV, 50D and 7D Mark II cameras and Canon EF 400 mm f5.6 and Canon $600 \mathrm{f} / 4$ lenses. While determining some species, binoculars were also used (Bushnell Powerview $12 \times 50 \mathrm{~mm}$ ). Especially while observing and photographing on riversides, camouflage was used.

Reptile and amphibian determination was made in the university campus and the immediate vicinity. Samples were caught by hand, and then identified.

Photo-traps (Bushnell Trophy Cam HD Max) were used in order to identify mammal species. This study does not include the determination of small mammal fauna with the use of phototraps. Mammal species captured by photo-traps were identified using the videos and photographs acquired by the traps. Photo-traps were mounted around the campus. The photo trap data were examined every two weeks. Extensive sources $[11,19,29-37]$ were utilized for the listing of the mammal species.

Visual Encounter Survey (VES) was used to determine the species in the Herpetofauna [38]. The VES is based on the systematic observation of animals at a specified time in the field study. This 
observation method is appropriate for assembling inventory and observation studies of the species in an aquatic or terrestrial habitat. The VES is used to determine species richness in a region, create a species list, and estimate the relative abundance of the species in a habitat. In the implementation of this method, observing species themselves by advancing in random transects in a particular field, or indirect observations such as slough and left traces are carried out. In this study, species lists were created by making direct observations in Ordu University campus and the immediate vicinity, in 2011 and in 2015 in fall and spring.

Species conservation status were organized according to the IUCN, BERN, and CITES $[39,40]$ criterias.

\section{RESULTS AND DISCUSSION}

A total of 168 bird species belonging to 50 families and 20 orders were listed from the city center and adjacent parts of Ordu (Table 1). Although two species from Columbidae (COLUMBIFORMES) family have been known to distribute in neighbor areas through Giresun, they were not able to be recorded in the study area. These are: Streptopelia turtur (European Turtle Dove) and S. decaocto (Collared Dove). Apus apus (Swift) and Tachymarptis melba (Alpine Swift) from Apodidae (APODIFORMES) family were registered in KuşBank [41] and recorded from Ordu, but were not included in the list since they cannot be verified. Similarly Cisticola juncidis (Fan-tailed Warbler) from Cisticolidae (PASSERIFORMES), mostly seen in our Mediterranean and Aegean coasts, was also excluded from the list.

Table 1. The list of birds of Ordu city.

\begin{tabular}{|c|c|c|c|c|c|}
\hline $\begin{array}{l}\text { ORDO Family } \\
\text { Species }\end{array}$ & Names of taxa & $\begin{array}{l}2014 \text { Feb } \\
\text { IUCN }\end{array}$ & $\begin{array}{l}2014 \text { Feb } \\
\text { BERN }\end{array}$ & $\begin{array}{l}2013 \text { June } \\
\text { CITES }\end{array}$ & 2013-2014 MAK \\
\hline GALLIFORMES & $\begin{array}{l}\text { Turkeys, grouse, } \\
\text { chickens, } \\
\text { pheasants }\end{array}$ & & & & \\
\hline Phasianidae & $\begin{array}{l}\text { Pheasants and } \\
\text { partridges }\end{array}$ & & & & \\
\hline Coturnix coturnix & Common quail & LC & III & & III \\
\hline ANSERIFORMES & $\begin{array}{l}\text { Screamers, } \\
\text { ducks, geese, } \\
\text { swans }\end{array}$ & & & & \\
\hline Anatidae & Ducks, Geese & & & & \\
\hline Anser albifrons & $\begin{array}{l}\text { Greater white- } \\
\text { fronted goose }\end{array}$ & LC & III & & III \\
\hline Cygnus olor & Mute swan & LC & III & & 1 \\
\hline Cygnus cygnus & Whooper swan & LC & ॥ & & I \\
\hline Tadorna ferruginea & Ruddy shelduck & LC & ॥ & & I \\
\hline Tadorna tadorna & $\begin{array}{l}\text { Common } \\
\text { shelduck }\end{array}$ & LC & ॥ & & I \\
\hline Anas penelope & $\begin{array}{l}\text { Eurasian } \\
\text { Wigeon }\end{array}$ & LC & III & & III \\
\hline Anas platyrhynchos & Mallard & LC & III & & III \\
\hline Anas clypeata & $\begin{array}{l}\text { Northern } \\
\text { shoveler }\end{array}$ & LC & III & & ॥ \\
\hline Anas acuta & Northern pintail & LC & III & & III \\
\hline
\end{tabular}


Table 1. The list of birds of Ordu city (Continue).

\begin{tabular}{|c|c|c|c|c|}
\hline Anas querquedula & Garganey & LC & III & III \\
\hline Anas crecca & Eurasian teal & LC & III & III \\
\hline Netta rufina & $\begin{array}{l}\text { Red-crested } \\
\text { pochard }\end{array}$ & LC & III & III \\
\hline Aythya ferina & $\begin{array}{l}\text { Common } \\
\text { pochard }\end{array}$ & LC & III & III \\
\hline Aythya nyroca & $\begin{array}{l}\text { Ferruginous } \\
\text { duck }\end{array}$ & NT & III & I \\
\hline Aythya fuligula & Tufted duck & LC & III & III \\
\hline Bucephala clangula & $\begin{array}{l}\text { Common } \\
\text { goldeneye }\end{array}$ & LC & II & III \\
\hline Mergellus albellus & Smew & LC & II & 1 \\
\hline Mergus serrator & $\begin{array}{l}\text { Red-breasted } \\
\text { merganser }\end{array}$ & LC & III & II \\
\hline GAVIIFORMES & Loons & & & \\
\hline Gaviidae & Loons & & & \\
\hline Gavia arctica & $\begin{array}{l}\text { Black-throated } \\
\text { loon }\end{array}$ & LC & ॥ & II \\
\hline PROCELLARIIFORMES & $\begin{array}{l}\text { Seabirds } \\
\text { (Petrels, } \\
\text { shearwaters,...) }\end{array}$ & & & \\
\hline Procellariidae & Shearwaters & & & \\
\hline Puffinus yelkouan & $\begin{array}{l}\text { Yelkouan } \\
\text { shearwater }\end{array}$ & VU & II & 1 \\
\hline PODICIPEDIFORMES & Grebe & & & \\
\hline Podicipedidae & Grebes & & & \\
\hline Tachybaptus ruficollis & Little grebe & LC & II & I \\
\hline Podiceps cristatus & $\begin{array}{l}\text { Great crested } \\
\text { grebe }\end{array}$ & LC & III & 1 \\
\hline Podiceps nigricollis & $\begin{array}{l}\text { Black-necked } \\
\text { grebe }\end{array}$ & LC & II & I \\
\hline CICONIIFORMES & Storks & & & \\
\hline Ciconiidae & Storks & & & \\
\hline Ciconia nigra & Black stork & LC & II & II \\
\hline Ciconia ciconia & White stork & LC & II & 1 \\
\hline PELECANIFORMES & $\begin{array}{l}\text { Ibises, } \\
\text { Spoonbills }\end{array}$ & & & \\
\hline Threskiornithidae & $\begin{array}{l}\text { Ibises, } \\
\text { Spoonbills }\end{array}$ & & & \\
\hline Plegadis falcinellus & Glossy ibis & LC & II & I \\
\hline Platalea leucorodia & $\begin{array}{l}\text { Eurasian } \\
\text { spoonbill }\end{array}$ & LC & II & II \\
\hline
\end{tabular}


52 | H. Sevgili et al. / Hacettepe J. Biol. \& Chem., 2016, 44 (1), 47-63

Table 1. The list of birds of Ordu city (Continue).

\begin{tabular}{|c|c|c|c|c|c|}
\hline Ardeidae & Herons, Bitterns & & & & \\
\hline Botaurus stellaris & Eurasian bittern & LC & II & & I \\
\hline Ixobrychus minutus & Little bittern & LC & II & & I \\
\hline Nycticorax nycticorax & $\begin{array}{l}\text { Black-crowned } \\
\text { night heron }\end{array}$ & LC & II & & I \\
\hline Ardeola ralloides & Squacco heron & LC & II & & 1 \\
\hline Bubulcus ibis & Cattle egret & LC & II & & I \\
\hline Ardea cinerea & Grey heron & LC & III & & II \\
\hline Ardea purpurea & Purple heron & LC & II & & I \\
\hline Casmerodius albus & $\begin{array}{l}\text { Great white } \\
\text { egret }\end{array}$ & LC & II & & 1 \\
\hline Egretta garzetta & Little egret & LC & II & & I \\
\hline Pelecanidae & Pelicans & & & & \\
\hline Pelecanus onocrotalus & $\begin{array}{l}\text { Great white } \\
\text { pelican }\end{array}$ & LC & II & & I \\
\hline Pelecanus crispus & $\begin{array}{l}\text { Dalmatian } \\
\text { pelican }\end{array}$ & VU & II & 1 & I \\
\hline SULIFORMES & $\begin{array}{l}\text { Cormorants, } \\
\text { Shags }\end{array}$ & & & & \\
\hline Phalacrocoracidae & Cormorants & & & & \\
\hline Phalacrocorax pygmeus & $\begin{array}{l}\text { Pygmy } \\
\text { cormorant }\end{array}$ & LC & II & & I \\
\hline Phalacrocorax carbo & Karabatak & LC & III & & II \\
\hline $\begin{array}{l}\text { Phalacrocorax } \\
\text { aristotelis }\end{array}$ & European shag & LC & II & & I \\
\hline FALCONIFORMES & Falcons & & & & \\
\hline Falconidae & Falcons & & & & \\
\hline Falco tinnunculus & Common kestrel & LC & II & II & I \\
\hline Falco subbuteo & Eurasian hobby & LC & II & II & I \\
\hline Falco peregrinus & Peregrine falcon & LC & II & I & I \\
\hline ACCIPITRIFORMES & $\begin{array}{l}\text { Hawks, Ospreys, } \\
\text { Eagles, } \\
\text { Buzzards }\end{array}$ & & & & \\
\hline Pandionidae & Ospreys & & & & \\
\hline Pandion haliaetus & Osprey & LC & ॥ & ॥ & I \\
\hline Accipitridae & Hawks & & & & \\
\hline Pernis apivorus & $\begin{array}{l}\text { European } \\
\text { honey-buzzard }\end{array}$ & LC & II & II & । \\
\hline Circaetus gallicus & $\begin{array}{l}\text { Short-toed } \\
\text { snake eagle }\end{array}$ & LC & II & II & I \\
\hline
\end{tabular}


Table 1. The list of birds of Ordu city (Continue).

\begin{tabular}{|c|c|c|c|c|c|}
\hline Circus aeruginosus & $\begin{array}{l}\text { Western marsh- } \\
\text { harrier }\end{array}$ & LC & II & II & I \\
\hline Circus cyaneus & Hen harrier & LC & II & II & I \\
\hline Accipiter nisus & $\begin{array}{l}\text { Eurasian } \\
\text { sparrohawk }\end{array}$ & LC & II & ॥ & 1 \\
\hline Buteo buteo & Buzzard & LC & II & II & I \\
\hline Buteo rufinus & $\begin{array}{l}\text { Long-legged } \\
\text { buzzard }\end{array}$ & LC & ॥ & ॥ & I \\
\hline Aquila pomarina & $\begin{array}{l}\text { Lesser spotted } \\
\text { eagle }\end{array}$ & LC & II & II & । \\
\hline Hieraaetus pennatus & Booted eagle & LC & II & II & 1 \\
\hline OTIDIFORMES & Bustard & & & & \\
\hline Otididae & Bustards & & & & \\
\hline Tetrax tetrax & Little bustard & NT & II & II & I \\
\hline GRUIFORMES & Crakes, Rails & & & & \\
\hline Rallidae & Rails and crakes & & & & \\
\hline Rallus aquaticus & Water rail & LC & III & & II \\
\hline Crex crex & Corn crake & LC & II & & I \\
\hline Porzana parva & Little crake & LC & II & & I \\
\hline Porzana porzana & Spotted crake & LC & II & & 1 \\
\hline Porphyrio porphyrio & $\begin{array}{l}\text { Purple } \\
\text { swamphen }\end{array}$ & LC & ॥ & & I \\
\hline Gallinula chloropus & $\begin{array}{l}\text { Common } \\
\text { moorhen }\end{array}$ & LC & III & & II \\
\hline Fulica atra & Eurasian coot & LC & III & & III \\
\hline Grus grus & Common crane & LC & II & II & I \\
\hline CHARADRIIFORMES & $\begin{array}{l}\text { Waders, gulls, } \\
\text { auks }\end{array}$ & & & & \\
\hline Haematopodidae & Oystercatchers & & & & \\
\hline Haematopus ostralegus & $\begin{array}{l}\text { Eurasian } \\
\text { oystercatcher }\end{array}$ & LC & III & & II \\
\hline Recurvirostridae & $\begin{array}{l}\text { Avocets and } \\
\text { stilts }\end{array}$ & & & & \\
\hline Himantopus himantopus & $\begin{array}{l}\text { Black-winged } \\
\text { stilt }\end{array}$ & LC & II & & I \\
\hline Charadriidae & $\begin{array}{l}\text { Plovers and } \\
\text { lapwings }\end{array}$ & & & & \\
\hline Vanellus vanellus & $\begin{array}{l}\text { Northern } \\
\text { lapwing }\end{array}$ & LC & III & & II \\
\hline Charadrius dubius & $\begin{array}{l}\text { Little ringed } \\
\text { plover }\end{array}$ & LC & II & & 1 \\
\hline
\end{tabular}


$54 \mid$ H. Sevgili et al. / Hacettepe J. Biol. \& Chem., 2016, 44 (1), 47-63

Table 1. The list of birds of Ordu city (Continue).

\begin{tabular}{|c|c|c|c|c|}
\hline Scolopacidae & $\begin{array}{l}\text { Snipe-like } \\
\text { waders }\end{array}$ & & & \\
\hline Gallinago gallinago & Common snipe & LC & III & III \\
\hline Limosa limosa & $\begin{array}{l}\text { Black-tailed } \\
\text { Godwit }\end{array}$ & NT & III & II \\
\hline Numenius phaeopus & Whimbrel & LC & III & II \\
\hline Tringa totanus & $\begin{array}{l}\text { Common } \\
\text { redshank }\end{array}$ & LC & III & II \\
\hline Tringa ochropus & Green sandpiper & LC & II & 1 \\
\hline Tringa glareola & Wood sandpiper & LC & II & 1 \\
\hline Actitis hypoleucos & $\begin{array}{l}\text { Common } \\
\text { sandpiper }\end{array}$ & LC & II & I \\
\hline Calidris alba & Sanderling & LC & II & I \\
\hline Calidris alpina & Dunlin & LC & II & I \\
\hline Laridae & Gulls & & & \\
\hline Larus canus & Common gull & LC & III & II \\
\hline Larus cachinnans & Caspian gull & LC & III & \\
\hline Larus michahellis & $\begin{array}{l}\text { Yellow legged } \\
\text { gull }\end{array}$ & LC & III & II \\
\hline Larus fuscus & $\begin{array}{l}\text { Lesser black- } \\
\text { backed gull }\end{array}$ & LC & & II \\
\hline Larus ichthyaetus & Pallas's gull & LC & III & II \\
\hline Larus ridibundus & $\begin{array}{l}\text { Black-headed } \\
\text { gull }\end{array}$ & LC & III & II \\
\hline Larus genei & $\begin{array}{l}\text { Slender-billed } \\
\text { gull }\end{array}$ & LC & II & I \\
\hline Larus melanocephalus & $\begin{array}{l}\text { Mediterranean } \\
\text { gull }\end{array}$ & LC & II & I \\
\hline Larus minutus & Little gull & LC & II & 1 \\
\hline Sternidae & Terns & & & \\
\hline Sterna caspia & Caspian tern & LC & II & I \\
\hline Sterna sandvicensis & Sanwich tern & LC & II & I \\
\hline Chlidonias hybrida & Whiskered tern & LC & II & I \\
\hline Chlidonias leucopterus & $\begin{array}{l}\text { White-winged } \\
\text { tern }\end{array}$ & LC & II & I \\
\hline Stercorariidae & Skuas & & & \\
\hline Stercorarius parasiticus & Parasitic jaeger & LC & III & II \\
\hline COLUMBIFORMES & Pigeons & & & \\
\hline Columbidae & Pigeons, Doves & & & \\
\hline Columba livia & Rock dove & LC & III & III \\
\hline
\end{tabular}


Table 1. The list of birds of Ordu city (Continue).

\begin{tabular}{|c|c|c|c|c|}
\hline $\begin{array}{l}\text { Stigmatopelia } \\
\text { senegalensis }\end{array}$ & Laughing dove & LC & III & II \\
\hline CUCULIFORMES & Cuckoos & & & \\
\hline Cuculidae & Cuckoos & & & \\
\hline Cuculus canorus & Common cuckoo & LC & III & I \\
\hline STRIGIFORMES & Owls & & & \\
\hline Strigidae & Owls & & & \\
\hline Strix aluco & Tawny owl & LC & II & II \\
\hline Athene noctua & Little owl & LC & II & II \\
\hline CORACIIFORMES & $\begin{array}{l}\text { Kingfishers, } \\
\text { Bee-eaters }\end{array}$ & & & \\
\hline Alcedinidae & Kingfishers & & & \\
\hline Alcedo atthis & $\begin{array}{l}\text { Common } \\
\text { kingfisher }\end{array}$ & LC & II & I \\
\hline Meropidae & Bea-eaters & & & \\
\hline Merops apiaster & $\begin{array}{l}\text { European bee- } \\
\text { eater }\end{array}$ & LC & II & I \\
\hline BUCEROTIFORMES & $\begin{array}{l}\text { Hornbills, } \\
\text { Hoopoes }\end{array}$ & & & \\
\hline Upupidae & Hoopoes & & & \\
\hline Upupa epops & $\begin{array}{l}\text { Common } \\
\text { hoopoe }\end{array}$ & LC & III & I \\
\hline PICIFORMES & Woodpeckers & & & \\
\hline Picidae & Woodpeckers & & & \\
\hline Jynx torquilla & $\begin{array}{l}\text { Eurasian } \\
\text { Wryneck }\end{array}$ & LC & II & I \\
\hline Dendrocopos medius & $\begin{array}{l}\text { Middle spotted } \\
\text { woodpecker }\end{array}$ & LC & II & I \\
\hline Dendrocopos syriacus & $\begin{array}{l}\text { Syrian } \\
\text { woodpecker }\end{array}$ & LC & II & I \\
\hline PASSERIFORMES & Songbirds & & & \\
\hline Laniidae & Shrikes & & & \\
\hline Lanius collurio & $\begin{array}{l}\text { Red-backed } \\
\text { shrike }\end{array}$ & LC & II & 1 \\
\hline Lanius nubicus & Masked shrike & LC & II & 1 \\
\hline Oriolidae & $\begin{array}{l}\text { Old World } \\
\text { orioles }\end{array}$ & & & \\
\hline Oriolus oriolus & $\begin{array}{l}\text { Eurasian golden } \\
\text { oriole }\end{array}$ & LC & II & I \\
\hline Corvidae & Crows & & & \\
\hline Garrulus glandarius & Eurasian jay & LC & & III \\
\hline
\end{tabular}


56 | H. Sevgili et al. / Hacettepe J. Biol. \& Chem., 2016, 44 (1), 47-63

Table 1. The list of birds of Ordu city (Continue).

\begin{tabular}{|c|c|c|c|c|}
\hline Corvus monedula & $\begin{array}{l}\text { Western } \\
\text { jackdaws }\end{array}$ & LC & & III \\
\hline Corvus frugilegus & Rook & LC & & III \\
\hline Corvus cornix & Hooded crow & UR & & III \\
\hline Corvus corax & Common raven & LC & III & II \\
\hline Paridae & Tits & & & \\
\hline Parus major & Great tit & LC & II & I \\
\hline Parus caeruleus & Eurasian blue tit & LC & II & I \\
\hline Hirundinidae & Swallows & & & \\
\hline Hirundo rustica & Barn swallow & LC & II & I \\
\hline Delichon urbicum & $\begin{array}{l}\text { Common house } \\
\text { martin }\end{array}$ & LC & $\|$ & 1 \\
\hline Aegithalidae & Long-tailed tits & & & \\
\hline Aegithalos caudatus & Long-tailed tit & LC & III & II \\
\hline Alaudidae & Larks & & & \\
\hline Galerida cristata & Crested lark & LC & III & ॥ \\
\hline Alauda arvensis & Eurasian skylark & LC & III & II \\
\hline Cettiidae & Bush warblers & & & \\
\hline Cettia cetti & Cetti's warbler & LC & III & I \\
\hline Acrocephalidae & Warblers & & & \\
\hline $\begin{array}{l}\text { Acrocephalus } \\
\text { schoenobaenus }\end{array}$ & Sedge warbler & LC & III & I \\
\hline $\begin{array}{l}\text { Acrocephalus } \\
\text { scirpaceus }\end{array}$ & $\begin{array}{l}\text { Eurasian reed } \\
\text { warbler }\end{array}$ & LC & III & 1 \\
\hline Acrocephalus palustris & Marsh warbler & LC & III & 1 \\
\hline $\begin{array}{l}\text { Acrocephalus } \\
\text { arundinaceus }\end{array}$ & $\begin{array}{l}\text { Great reed } \\
\text { warbler }\end{array}$ & LC & III & I \\
\hline Phylloscopidae & Willow warblers & & & \\
\hline Phylloscopus trochilus & Willow warbler & LC & III & 1 \\
\hline Phylloscopus collybita & $\begin{array}{l}\text { Common } \\
\text { chiffchaff }\end{array}$ & LC & III & I \\
\hline Phylloscopus sibilatrix & Wood warbler & LC & III & 1 \\
\hline Sylviidae & $\begin{array}{l}\text { True warblers } \\
\text { and parrotbills }\end{array}$ & & & \\
\hline Sylvia atricapilla & $\begin{array}{l}\text { Eurasian } \\
\text { blackcap }\end{array}$ & LC & $\|$ & 1 \\
\hline Sylvia borin & Garden warbler & LC & ॥ & I \\
\hline Sylvia communis & $\begin{array}{l}\text { Common } \\
\text { whitethroat }\end{array}$ & LC & II & I \\
\hline Sylvia curruca & $\begin{array}{l}\text { Lesser } \\
\text { whitethroat }\end{array}$ & LC & ॥ & 1 \\
\hline
\end{tabular}


Table 1. The list of birds of Ordu city (Continue).

\begin{tabular}{|c|c|c|c|c|}
\hline Sylvia nisoria & Barred warbler & LC & II & 1 \\
\hline Sylvia melanocephala & $\begin{array}{l}\text { Sardininan } \\
\text { warbler }\end{array}$ & LC & II & I \\
\hline Regulidae & Kinglets, Crests & & & \\
\hline Regulus regulus & Goldcrest & LC & II & 1 \\
\hline Regulus ignicapilla & $\begin{array}{l}\text { Common } \\
\text { firecast }\end{array}$ & LC & II & I \\
\hline Troglodytidae & Wrens & & & \\
\hline Troglodytes troglodytes & Eurasien wren & LC & II & I \\
\hline Sturnidae & Starlings & & & \\
\hline Sturnus vulgaris & $\begin{array}{l}\text { Common } \\
\text { starling }\end{array}$ & LC & & II \\
\hline Turdidae & Thrushes & & & \\
\hline Turdus merula & $\begin{array}{l}\text { Common } \\
\text { blackbird }\end{array}$ & LC & III & III \\
\hline Turdus philomelos & Song thrush & LC & III & II \\
\hline Muscicapidae & Flycatchers & & & \\
\hline Erithacus rubecula & European robin & LC & II & 1 \\
\hline Luscinia Iuscinia & $\begin{array}{l}\text { Thrush } \\
\text { nightingale }\end{array}$ & LC & II & I \\
\hline Luscinia svecica & Bluethroat & LC & II & I \\
\hline Phoenicurus ochruros & Black redstart & LC & II & I \\
\hline $\begin{array}{l}\text { Phoenicurus } \\
\text { phoenicurus }\end{array}$ & $\begin{array}{l}\text { Common } \\
\text { redstart }\end{array}$ & LC & II & 1 \\
\hline Saxicola rubetra & Whinchat & LC & II & I \\
\hline Saxicola torquatus & $\begin{array}{l}\text { Common } \\
\text { stonechat }\end{array}$ & LC & II & I \\
\hline Saxicola maurus & $\begin{array}{l}\text { Siberian } \\
\text { stonechat }\end{array}$ & NR & III & \\
\hline Oenanthe oenanthe & Wheatear & LC & II & I \\
\hline Oenanthe isabellina & $\begin{array}{l}\text { Isabelline } \\
\text { wheatear }\end{array}$ & LC & II & 1 \\
\hline Muscicapa striata & $\begin{array}{l}\text { Spotted } \\
\text { flycatcher }\end{array}$ & LC & II & I \\
\hline Ficedula parva & $\begin{array}{l}\text { Red-breasted } \\
\text { flycatcher }\end{array}$ & LC & II & 1 \\
\hline Cinclidae & Dippers & & & \\
\hline Cinclus cinclus & $\begin{array}{l}\text { White-throated } \\
\text { dipper }\end{array}$ & LC & II & 1 \\
\hline Passeridae & Sparrows & & & \\
\hline Passer domesticus & House sparrow & LC & & III \\
\hline
\end{tabular}


58 H. Sevgili et al. / Hacettepe J. Biol. \& Chem., 2016, 44 (1), 47-63

Table 1. The list of birds of Ordu city (Continue).

\begin{tabular}{|c|c|c|c|c|}
\hline Passer montanus & $\begin{array}{l}\text { Eurasian tree } \\
\text { sparrow }\end{array}$ & LC & III & II \\
\hline Prunellidae & Accentors & & & \\
\hline Prunella modularis & Dunnock & LC & III & I \\
\hline Motacillidae & Wagtails, Pipits & & & \\
\hline Motacilla alba & White wagtail & LC & III & I \\
\hline Motacilla citreola & Citrine wagtail & LC & III & I \\
\hline Motacilla flava & Yellow wagtail & LC & II & I \\
\hline Motacilla cinerea & Grey wagtail & LC & II & I \\
\hline Anthus pratensis & Meadow pipit & LC & II & I \\
\hline Anthus spinoletta & Water pipit & LC & II & I \\
\hline Fringillidae & Finches & & & \\
\hline Fringilla coelebs & $\begin{array}{l}\text { Common } \\
\text { chaffinch }\end{array}$ & LC & III & ॥ \\
\hline Carduelis chloris & $\begin{array}{l}\text { European } \\
\text { greenfinch }\end{array}$ & LC & III & । \\
\hline Carduelis spinus & Eurasian siskin & LC & III & I \\
\hline Carduelis carduelis & Goldfinch & LC & III & I \\
\hline Carduelis cannabina & Common linnet & LC & III & I \\
\hline Emberizidae & Buntings & & & \\
\hline Miliaria calandra & Corn bunting & LC & III & II \\
\hline Emberiza cia & Rock bunting & LC & III & I \\
\hline Emberiza rustica & Rustic bunting & LC & III & I \\
\hline Emberiza schoeniclus & Reed bunting & LC & III & I \\
\hline
\end{tabular}

Table 2. The list of the mammals of Ordu city.

\begin{tabular}{|c|c|c|c|c|}
\hline $\begin{array}{l}\text { ORDO } \\
\text { Family } \\
\text { Species }\end{array}$ & Name of taxa & IUCN & BERN & CITES \\
\hline ERINACEOMORPHA & Hedgehogs & & & \\
\hline Erinaceidae & Hedgehogs & & & \\
\hline Erinaceus concolor & White-chested hedgehog & LC & & \\
\hline SORICOMORPHA & Shrew & & & \\
\hline Soricidae & Shrews & & & \\
\hline Sorex raddei & Radde's shrew & LC & III & \\
\hline Neomys teres & $\begin{array}{l}\text { Transcaucasian water } \\
\text { shrew }\end{array}$ & LC & III & \\
\hline $\begin{array}{l}\text { Crocidura } \\
\text { gueldenstaedtii }\end{array}$ & Lesser shrew & NE & III & \\
\hline
\end{tabular}


Table 2. The list of the mammals of Ordu city (Continue).

\begin{tabular}{|c|c|c|c|}
\hline Crocidura suaveolens & $\begin{array}{l}\text { Lesser white-toothed } \\
\text { shrew }\end{array}$ & LC & III \\
\hline Talpidae & Moles & & \\
\hline Talpa levantis & Levant mole & LC & \\
\hline CHIROPTERA & Bats & & \\
\hline Rhinolophidae & Horseshoe bats & & \\
\hline $\begin{array}{l}\text { Rhinolophus } \\
\text { ferrumequinum }\end{array}$ & Gretaer horseshoe bats & LC & II \\
\hline $\begin{array}{l}\text { Rhinolophus } \\
\text { hipposideros }\end{array}$ & Lesser horseshoe bats & LC & II \\
\hline Rhinolophus euryale & $\begin{array}{l}\text { Mediterranean } \\
\text { horseshoe bat }\end{array}$ & NT & II \\
\hline Vespertilionidae & Vesper bats & & \\
\hline Myotis myotis & Greater mouse-eared bat & LC & II \\
\hline Myotis blythii & Lesser mouse-eared bat & LC & II \\
\hline Nyctalus leisleri & Lesser noctule & LC & II \\
\hline Eptesicus serotinus $^{1}$ & Serotine bat & LC & II \\
\hline Pipistrellus pipistrellus & Common pipistrelle & LC & III \\
\hline Miniopteridae & Long-winged bats & & \\
\hline Miniopterus schreibersii & Common bent-wing bag & NT & II \\
\hline LAGOMORPHA & Lagomorphs & & \\
\hline Leporidae & Hares & & \\
\hline Lepus europaeus & $\begin{array}{l}\text { Euopean hare/Brown } \\
\text { hare }\end{array}$ & LC & III \\
\hline RODENTIA & Rodents & & \\
\hline Sciuridae & Squirrels & & \\
\hline Sciurus anomalus & Caucasian squirrel & LC & II \\
\hline Cricetidae & Voles, hamsters & & \\
\hline Cricetulus migratorius & Gray dwarf hamster & LC & \\
\hline Myodes glareolus & Bank vole & LC & \\
\hline Microtus levis & Southern vole & LC & \\
\hline Microtus subterraneus & Common pine vole & LC & \\
\hline Microtus majori² & Major's pine vole & LC & \\
\hline Muridae & Mouse, Rats & & \\
\hline Apodemus mystacinus & $\begin{array}{l}\text { Broad-toothed field } \\
\text { mouse }\end{array}$ & LC & \\
\hline Apodemus flavicollis & Yellow-necked mouse & LC & \\
\hline Apodemus witherbyi & Steppe field mouse & LC & \\
\hline Rattus rattus & Black rat & LC & \\
\hline
\end{tabular}


60 H. Sevgili et al. / Hacettepe J. Biol. \& Chem., 2016, 44 (1), 47-63

Table 2. The list of the mammals of Ordu city (Continue).

\begin{tabular}{|c|c|c|c|c|}
\hline Rattus norvegicus & Brown rat & LC & & \\
\hline $\begin{array}{l}\text { Mus musculus } \\
\text { domesticus }\end{array}$ & House mouse & LC & & \\
\hline Mus macedonicus & Macedonian mouse & LC & & \\
\hline Gliridae & Dormouses & & & \\
\hline Dryomys nitedula ${ }^{3}$ & Forest dormouse & LC & III & \\
\hline $\begin{array}{l}\text { Muscardinus } \\
\text { avellanarius }\end{array}$ & Hazel dormouse & LC & III & \\
\hline CARNIVORA & Carnivore & & & \\
\hline Canidae & Foxes, Jackals & & & \\
\hline Canis aureus & Golden jackal & LC & & \\
\hline Vulpes vulpes & Red fox & LC & & \\
\hline Mustelidae & Martens, Weasels & & & \\
\hline Mustela nivalis & Least weasel & LC & III & \\
\hline Martes martes & European pine marten & LC & III & \\
\hline Martes foina & Stone marten & LC & III & \\
\hline Lutra lutra ${ }^{4}$ & Lutra & NT & II & 1 \\
\hline Felidae & Kedigiller & & & \\
\hline Felis silvestris & Yaban Kedisi & LC & II & II \\
\hline ARTIODACTYLA & Even-toed ungulate & & & \\
\hline Suidae & Pigs, Boars & & & \\
\hline Sus scrofa & Wild boar & LC & & \\
\hline Cervidae & Deers & & & \\
\hline Capreolus capreolus & Roe deer & LC & III & \\
\hline
\end{tabular}

'Recorded from Bulancak (Giresun), very close to Ordu city (A. Karataş, unpublished data), therefore this species is added in to the list.

${ }^{2}$ Although there is no record from Ordu city, the species is known from Ulubey town, very close to Ordu [43, cf. 44]. Thus, we added the species to the current list of the city.

${ }^{3}$ It is recorded from Bulancak (Giresun) (A. Karataş, unpublished data).

${ }^{4} \mathrm{~L}$. lutra, we did not find from Ordu although it is observed from two different localities in Bulancak (Giresun) (A. Karataş, unpublished data).

Table 3. Species list of the Herpetofauna of Ordu city.

\begin{tabular}{|c|c|c|c|c|}
\hline $\begin{array}{l}\text { ORDO } \\
\text { Family } \\
\text { Species }\end{array}$ & Name of taxa & IUCN & BERN & CITES \\
\hline SQUAMATA & Scaled reptiles & & & \\
\hline Anguidae & Glass lizards & & & \\
\hline Anguis fragilis & Slow worm, Blindworm & & III & \\
\hline Lacertidae & Lizards & & & \\
\hline Lacerta viridis & Green lizard & LC & II & \\
\hline Darevskia rudis & Spiny-tailed lizard & LC & III & \\
\hline SERPENTES & Snakes & & & \\
\hline
\end{tabular}


Table 3. Species list of the Herpetofauna of Ordu city (Continue).

\begin{tabular}{|c|c|c|c|c|}
\hline Colubridae & Colubrids & & & \\
\hline Coronella austriaca & Smooth snake & & II & \\
\hline Natrix natrix & Grass snake & LC & III & \\
\hline Elaphe Iongissima & Aesculapian snake & LC & II & \\
\hline TESTUDINATA & Turtles & & & \\
\hline Emydidae & Pond/Mars turtles & & & \\
\hline Emys orbicularis & European pond turtle & NT & II & \\
\hline URODELA & Newts and Salamanders & & & \\
\hline Salamandridae & Salamenders & & & \\
\hline Triturus ivanbureschi & $\begin{array}{l}\text { Balkan-Anatolian } \\
\text { crested newt }\end{array}$ & LC & ॥ & \\
\hline ANURA & Frogs & & & \\
\hline Hylidae & Tree frogs & & & \\
\hline Hyla orientalis & $\begin{array}{l}\text { Oriental tree frog, } \\
\text { Green frog }\end{array}$ & & III & \\
\hline Ranidae & True frogs & & & \\
\hline Rana dalmatina & Agile frog & LC & III & \\
\hline Pelophylax ridibundus & Marsh frog & LC & III & - \\
\hline
\end{tabular}

40 species of mammals belonging to 16 families and 7 orders were listed (Table 2 ). Despite a few of these species were not recorded in the study area, they were included in the list because of their high possibility of existence in the area for distributing in adjacent parts and sharing almost the same habitat type (see footnotes in Table 3). In addition, some species like Microtus socialis (Social vole, Doğu Kırfaresi) given from inner parts as from Gölköy and Mesudiye were also not included in the list. These districts are located in the Central Anatolia border of Ordu and are different from the study area which shows boreal characteristics in both ecological and zoogeographical terms.

On the other hand, Monachus monachus (Mediterranean monk seal) has been regarded as extinct (RE) in all the Black Sea after been observed $(N=1)$ in the early 1980 [42] in Perşembe. Ursus arctos (Brown Bear) and Cervus elaphus (Red Deer) records which were given by Kumerloeve [35], has not been verified by the recent observations and were not added to the list since they are assessed as extinct in Ordu (central district). Similarly, Rupicapra rupicapra (Chamois) records given from Ordu by the same author are likely to be misidentification of Capra aegagrus (Wild Goat). Indeed, this species is mostly distributed in the high mountains of the Eastern Black Sea.

A total of 17 herptile species, including 3 salamanders (Urodela), 6 frogs (Anura), 4 lizards and 4 snakes were recorded according to the results of the only herpetofaunistic study carried out in Ordu and Giresun which also covers the present study area [27]. However in this study, a total of 11 species were identified, including one salamanders (Urodela), 3 frogs (Anura), one turtle, 3 lizards and 3 snakes (Table 3 ).

\section{ACKNOWLEDGEMENTS}

Funding was provided by Ordu University, Turkey (Scientific Research Projects Coordination Unit, Project no: AR-1223). We would likte to thank Ömer Furtun, Ömer Kulaçoğlu, Mehmet Özcan, Arslan Kezer, Dr. Kiraz Erciyas and Nizamettin Yavuz providing some their field observation data. 


\section{References}

1. Anonim. United Nations, http://www.un.org Accessed 27.10.2015.

2. Anonim. Türkiye İstatistik Kurumu, http://www.tuik. gov.tr Accessed 27.10.2015.

3. J.R. Miller, Biodiversity conservation and the extinction of experience, Trends Ecol. Evol., 20 (2005) 430-434.

4. K. Szlavecz, P. Warren, S. Pickett, Biodiversity on the Urban Landscape. USA, Springer-Verlag Berlin Heidelberg. (2011), Number of 75-101 p.

5. M.L. McKinney, Urbanization, biodiversity, and conservation, Bioscience, 52 (2002) 883-890.

6. C.H. Sekercioglu, S. Anderson, E. Akcay, R. Bilgin, O.E. Can, G. Semiz, C. Tavsanoglu, M.B. Yokes, A. Soyumert, K. Ipekdal, I.K. Saglam, M. Yucel, H.N. Dalfes, Turkey's globally important biodiversity in crisis, Biological Conservation, 144 (2011) 2752-2769.

7. N. Demir, Ordu ilinin eski adı "Kotyora" ve tarihi alt yapısı, Turkish Studies/Türkoloji Araştırmaları, 2 (2007) 179-184.

8. S. Trak, Giresun-Ordu ve ardülkesinde beşeri coğrafya araştırmaları, Ankara Üniversitesi Dil ve Tarih-Coğrafya Fakültesi Dergisi, 1 (1943) 145-155.

9. G. Gürgen, PhD, Ankara Üniversitesi, Sosyal Bilimler Enstitüsü, Doktora tezi, Ankara, 1993.

10. Anonim. TRAKUŞ (Türkiye'nin Anonim Kuşları), http:// www.trakus.org Accessed 01.12.2015.

11. Anonim. TRAMEM (Türkiye'nin Anonim Memelileri), http://www.tramem.org Accessed 01.12.2015.

12. G.M. Kirwan, R.P. Martins, G. Eken, P. Davidson, A Checklist of the Birds of Turkey, Sandgrouse, Supplement, 1 (1998) 1-29.

13. C. Bilgin, PhD Thesis, METU, Ankara (1994).

14. G.M. Kirwan, K.A. Boyla, P. Castell, B. Demirci, M. Özen, H. Welch, T. Marlow. The Birds of Turkey. Christopher Helm., London, UK, 2008.

15. Anonim. Doğal Hayat, http://www.dogalhayat.org Accessed 28.11.2015.

16. S. Barış, Ö. Sağlam, K. Erciyas, N. Yavuz, A.C. Özsemir. Önemli bir doğa mirası: Kızılırmak Deltası kuşları. Doğa ve Yaban Hayatı Koruma Derneği (DYDK), Samsun, 2010.

17. T. Göktürk, T. Artvinli, F. Bucak, Artvin kuş faunası, Artvin Çoruh Üniversitesi, Orman Fakültesi Dergisi, 9 (2008) 33-43.

18. S. Aydın, U. Duman, B. Gönül, A. Kır, A.B. Bağran. 2004 yılı Ordu il çevre durum raporu. T. C. Ordu Valiliği II Çevre ve Orman Müdürlüğü, 183 pp, Ordu, 2005.

19. B. Krystufek, V. Vohralik. Mammals of Turkey and Cyprus: Introduction, Checklist, Insectivora. Knjiznica Annales Majora, Koper, Slovenia, 2001.

20. N.R. Villasenor, D.A. Driscoll, M.A.H. Escobar, P. Gibbons, D. B. Lindenmayer, Urbanization Impacts on Mammals across Urban-Forest Edges and a Predictive Model of Edge Effects, Plos One, 9 (2014).

21. R. Lopucki, I. Mroz, L. Berlinski, M. Burzych, Effects of urbanization on small-mammal communities and the population structure of synurbic species: an example of a medium-sized city, Can. J. Zool., 91 (2013) 554561.
22. A.J. Hamer, M.J. McDonnell, The response of herpetofauna to urbanization: Inferring patterns of persistence from wildlife databases, Austral. Ecol., 35, (2010) 568-580.

23. T.J.C. Beebee, R.A. Griffiths, The amphibian decline crisis: A watershed for conservation biology?, Biological Conservation, 125 (2005) 271-285.

24. I. Baran. Türkiye Amfibi ve Sürüngenleri. TÜBiTAK Popüler Bilim Kitapları, 207, Ankara. 2008.

25. Anonim. AdaMerOs Herptil Türkiye: Türkiye Kurbağa ve Sürüngenleri Gözlemciliği ve Fotoğrafçılığı Topluluğu, http://www.turkherptil.org Accessed 02.12.2015.

26. I. Baran, M. Tosunoğlu, U. Kaya, Y. Kumlutaş, On the Herpetofauna of the Vicinity of Çamlıhemşin, Turkis Journal of Zoology, 21 (1997) 399-408.

27. Y. Kumlutaş, V. Tok, O. Türkozan, The Herpetofauna of the Ordu Giresun Region, Turkish Journal of Zoology, 22 (1998) 199-201.

28. I. Baran, I. Yılmaz, R. Kete, Y. Kumlutaş, H. Durmuş, Herpetofauna of west and middle Black Sea region, Turkish Journal of Zoology, 16 (1992) 275-288.

29. A. Karataş. Türkiye Yarasaları. Niğde Universitesi, BAP Birimi, No: 01.FEB.09, Niğde, 2009.

30. A. Karataş, M. Sözen, Contribution to karyology, distribution and taxonomic status of the Longwinged Bat, Miniopterus schreibersii (Chiroptera: Vespertilionidae), in Turkey, Zoology in the Middle East, 33 (2004) 51-64.

31. A. Karataş, M. Sözen, Karyology of three Vespertilionid bats (Chiroptera: Vespertilionidae) from Turkey, Acta Zoologica Academiae Scientarium Hungaricae, 53 (2007) 185-192.

32. B. Krystufek, V. Vohralik. Mammals of Turkey and Cyprus: Introduction Checklist Insectivora. Koper, 2001.

33. B. Krystufek, V. Vohralik. Mammals of Turkey and Cyprus: Rodentia I: Scuidae, Dipodidae, Gliridae, Arvicolinae. Koper, Zgodovinsko drustvo za juzno Primorsko Znanstveno-raziskovalno sredisce Republike Slovenije. 2005.

34. H. Kumerloeve, Zur Verbreitung Kleinasiatischer Raub- und Huftiere sowie eineger Großnager, Sonderdruck aus Säugetierkd. Mitt., 15 (1967) 337409.

35. H. Kumerloeve, Die Saugetierte (Mammalia) der Turkei, Veröff. Zool. Staatssamlung München., 18 (1975) 69-158.

36. F. Spitzenberger, Zur Verbreitung und Systematik türkischer Crocidurinae (Insectivora, Mammalia), Ann. Naturhistor. Mus. Wien, 74 (1968) 233-252.

37. N. Yiğit, E. Çolak, M. Sözen, A. Karataş. Rodents of Türkiye. Meteksan, Ankara, 2006.

38. M.L. Crump, NJ. Scott, Visual Encounter Surveys. Washington, Smithsonian Inst. Press., (1994), Number of $84-92 \mathrm{p}$

39. Anonim. IUCN Red List, http://www.iucnredlist.org Accessed 25.20.2015.

40. Anonim. CITES, http://www.cites.org Accessed 11.10.2015.

41. Anonim. Kuşbank, www.kusbank.org Accessed 01.12.2015.

42. B. Mursaloğlu, How to save the Monk seal, Communications Faculty of Science University of Ankara, Series C: Biology, 6 (1988) 227-233. 
43. F. Spitzenberger, H. Steiner, Über Insectenfresser (Insectivora) und Wühlmause (Microtinae) der Nordosttürkischen feuchtwalder, Bonner Zoological Bulletin, 4 (1962) 284-310.
44. M. Çağlar, Türkiye'nin gömülgen fareleri (Microtin), Türk Biyoloji Dergisi, 17 (1967) 103-118. 
